\title{
Validation of an Automated and Adjustable Blood Pressure System for Use with a Public Health Station
}

This article was published in the following Dove Press journal:

Vascular Health and Risk Management

\author{
Timothy R Macaulay (ID) \\ David N Erceg ${ }^{1,2}$ \\ Bjorn McMillan $\mathbb{D}^{2}$ \\ Joel E Ramirez' \\ Jesus F Dominguez' \\ Alberto F Vallejo' \\ E Todd Schroeder' \\ 'Division of Biokinesiology and Physical \\ Therapy, University of Southern California, \\ Los Angeles, CA, USA; ${ }^{2}$ Stayhealthy Inc., \\ Monrovia, CA, USA
}

\begin{abstract}
Objective: A new automated and adjustable blood pressure (BP) system has been developed to improve the accuracy of BP measurements on public-use health stations. This self-fitting BP system includes a mechanical cuff that wraps down to the user's arm prior to bladder inflation. The purpose of this study was to validate the adaptable BP system (ABPS) using the current standards from the Association for the Advancement of Medical Instrumentation (AAMI).
\end{abstract}

Methods: The AAMI/ISO 81060:2013 standards for clinical validation of non-invasive automated arterial BP measurement devices were followed precisely using the same arm sequential method. For each participant, BP was measured over multiple trials by trained observers alternating a reference sphygmomanometer with the ABPS. All study requirements were met with 85 qualifying participants, each with 3 valid paired determinations.

Results: The mean difference between ABPS BP and reference BP using all 255 paired determinations was $-2.4 \pm 7.7 \mathrm{mmHg}$ for systolic and $1.7 \pm 5.7 \mathrm{mmHg}$ for diastolic. The standard deviation of the averaged paired determinations per participant was $6.3 \mathrm{mmHg}$ for systolic and $5.2 \mathrm{mmHg}$ for diastolic. Arm circumference measurements had a mean error of $-2.1 \pm 2.4 \mathrm{~cm}\left(\mathrm{R}^{2}=0.87\right)$. A new prediction model for arm circumference was validated using a holdout dataset $\left(\mathrm{R}^{2}=0.94\right)$.

Conclusion: The results of the study confirm that the ABPS met all benchmarks established by the AAMI. The device accurately measures BP across a wide range of arm circumferences $(24-44 \mathrm{~cm})$ and is suitable for use by individuals to self-monitor BP.

Keywords: hypertension, cardiovascular disease, self-monitoring, systolic, diastolic, sphygmomanometer, cuff

\section{Introduction}

Hypertension is the leading modifiable risk factor for cardiovascular disease (CVD) and all-cause mortality. ${ }^{1,2}$ Evidence suggests that the gradient of increased CVD risk with progressively elevated blood pressure (BP) starts at lower values than previously defined. ${ }^{3}$ Despite an increasing public awareness of the potential consequences of elevated BP, reports continue to suggest that hypertension remains largely undiagnosed. ${ }^{4,5}$ To establish expert consensus, the American College of Cardiology and the American Heart Association (ACC/AHA) appraised systematic evaluations of recent trials examining $\mathrm{BP}$ and published the 2017 Guideline for the Prevention, Detection, Evaluation, and Management of High Blood Pressure in Adults. ${ }^{6}$ Compared with the previous report by Joint National Committee (JNC), the 2017 ACC/AHA guideline updated recommendations to define hypertension using lower systolic $(\geq 130 \mathrm{mmHg})$ and diastolic $(\geq 80 \mathrm{mmHg})$ BP levels. ${ }^{6}$ These new standards
Correspondence: Timothy R Macaulay Division of Biokinesiology and Physical Therapy, University of Southern

California, I540 E. Alcazar Street, CHP

149, Los Angeles, CA 90089, USA

$\mathrm{Tel}+$ I $408-332-3900$

Fax +323-442-15I5

Email tmacaula@usc.edu 
effectively increased hypertension prevalence from $32 \%$ to $46 \%$ and advise more intensive BP lowering for millions of United States (US) adults through both antihypertensive medication and nonpharmacological intervention. ${ }^{7}$

With the increased emphasis on BP monitoring, there is high demand for accessible and accurate self-screening for the detection and management of hypertension outside traditional health-care settings. ${ }^{8}$ Automated kiosk BP cuffs can improve self-monitoring by providing a means for patients to measure their BP outside of the clinic, without the need to purchase or operate a personal monitor. These kiosks play a major public health role with approximately 1000 uses/ month in a typical community-based pharmacy. ${ }^{9}$ However, the accuracy of these cuffs has been questioned, ${ }^{10}$ especially given their limited arm circumference ranges. Clearance from the US Food and Drug Administration (FDA) does not ensure that readings are accurate enough to be utilized by all in the clinical management of the disease. ${ }^{11}$ If cuff size is too small, it may lead to an inaccurately elevated BP reading while too large of a cuff may lead to a low or high reading. ${ }^{12}$ To ensure that measurements in the designated cuff size range for fixed kiosks are accurate, partnerships between independent third parties (eg, academia) and the equipment manufacturer are essential to validate this technology. Validation studies (such as reported here) following the International Organization for Standardization (ISO) provide the gold standard for the assessment and validation of measurement accuracy.
To address concerns for accommodating larger arm size in patients with higher risk of hypertension, an adaptable BP system (ABPS) has been developed, ${ }^{13}$ designed to fit the user's arm like standard health-care office protocol. A visual diagram of the ABPS is presented in Figure 1, including the new mechanical cuff on a sit-down health kiosk. The cuff is equipped with an adapted knuckle support assembly that provides four degrees freedom of movement for postural positioning and a fully automated motorized gearhead that wraps the cuff down to the user's arm prior to bladder inflation. This mechanism also provides a measure of arm circumference. The purpose of this study was to validate the ABPS using the ANSI/AAMI/ISO 81060-2:2013 standards ${ }^{14}$ and to assess the accuracy of arm circumference measurements.

\section{Methods \\ Participants}

This study was conducted at the following locations: University of Southern California (USC, Los Angeles, CA) Health Sciences Campus, USC Alhambra Campus (Alhambra, CA), South Pasadena Senior Center (South Pasadena, CA), and Pasadena Department of Public Health (Pasadena, CA). Requirements for same arm sequential BP testing were followed precisely as detailed in the ANSI/AAMI/ISO 81060-2:2013 standard. ${ }^{14}$ The ABPS was under the investigational stage as part of the 81060-2:2013 ANSI/AAMI/ISO device requirements for FDA submission purposes. A flow

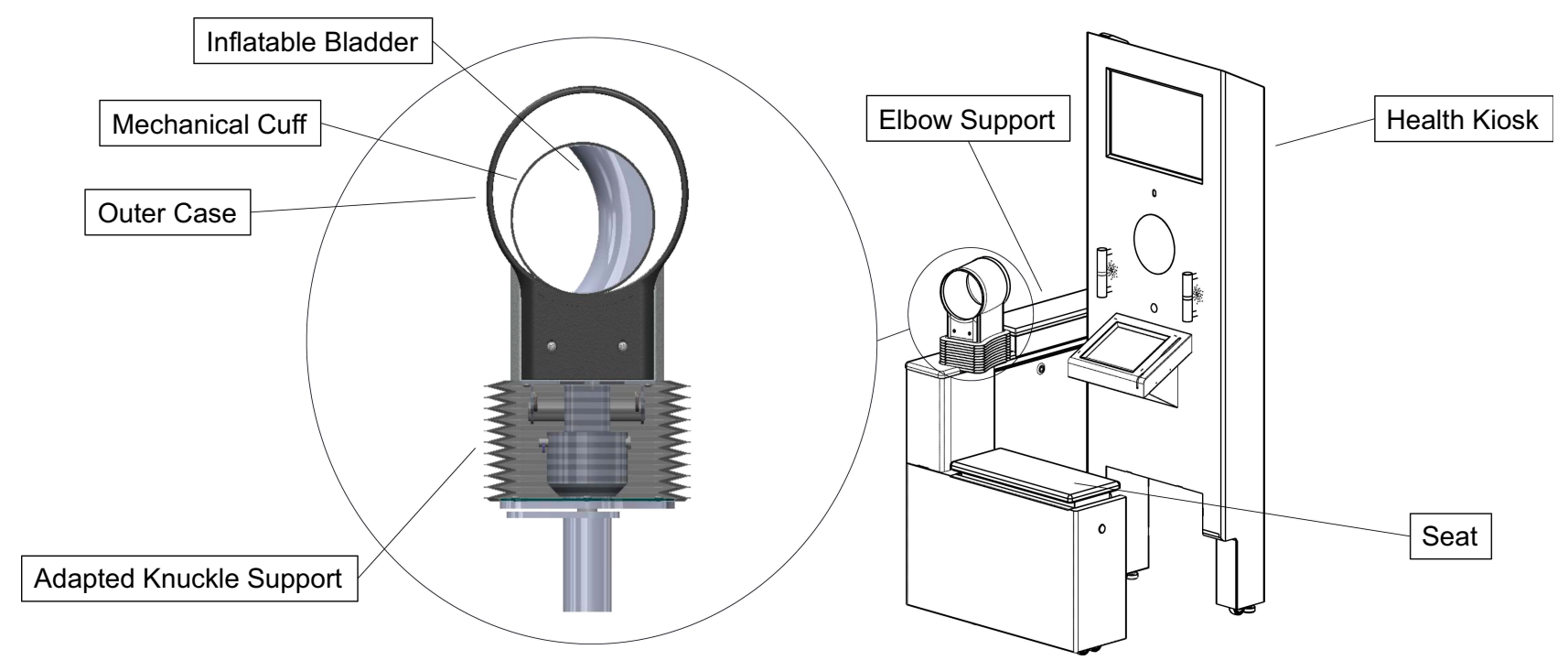

Figure I Schematic diagram of the adaptable blood pressure system. For blood pressure assessments, participants sit upright on the health kiosk facing the screen and insert their left arm through the mechanical cuff, resting their elbow and forearm on the support. The adapted knuckle support provides four degrees freedom of movement for comfortable postural positioning. Upon initiation, a motorized gearhead wraps the mechanical cuff down to the user's arm prior to bladder inflation. The cuff then operates like a normal automated blood pressure system, detecting blood pressure pulses as it deflates. Once the BP assessment is completed, the cuff fully deflates and expands, returning to the circumference of the starting position. 
diagram of participant recruitment and testing is shown in Figure 2. A total of 231 volunteer participants were tested to reach the minimum 85 eligible participants qualifying for statistical analyses. In brief, the standards require that study participants maintain a stable BP and meet the characteristic distributions provided in Table 1.

The following inclusion/exclusion criteria were used to determine eligibility: participants were included if they were between 12 and 92 years of age and healthy, but could be taking medication for BP regulation; participants were excluded if they had atrial or ventricular arrhythmias, used a pacemaker to maintain suitable heart rate, were missing their natural left arm, exhibited a musculoskeletal disorder that may prevent proper BP readings, or were considered special populations (eg, pregnant women). This study was approved by the USC Health Sciences Review Board, registered with ClinicalTrials.gov (ID: NCT03566888), and conducted in accordance with the Declaration of Helsinki. All adult participants provided written informed consent. Participants under the age of 18 years provided written informed assent to be in research and a parent or legal guardian provided written informed consent.

After obtaining written informed consent, participant anthropometrics were recorded: height, weight, and left arm circumference. Participants were asked to remove clothing that covered the arm location where the BP cuffs were

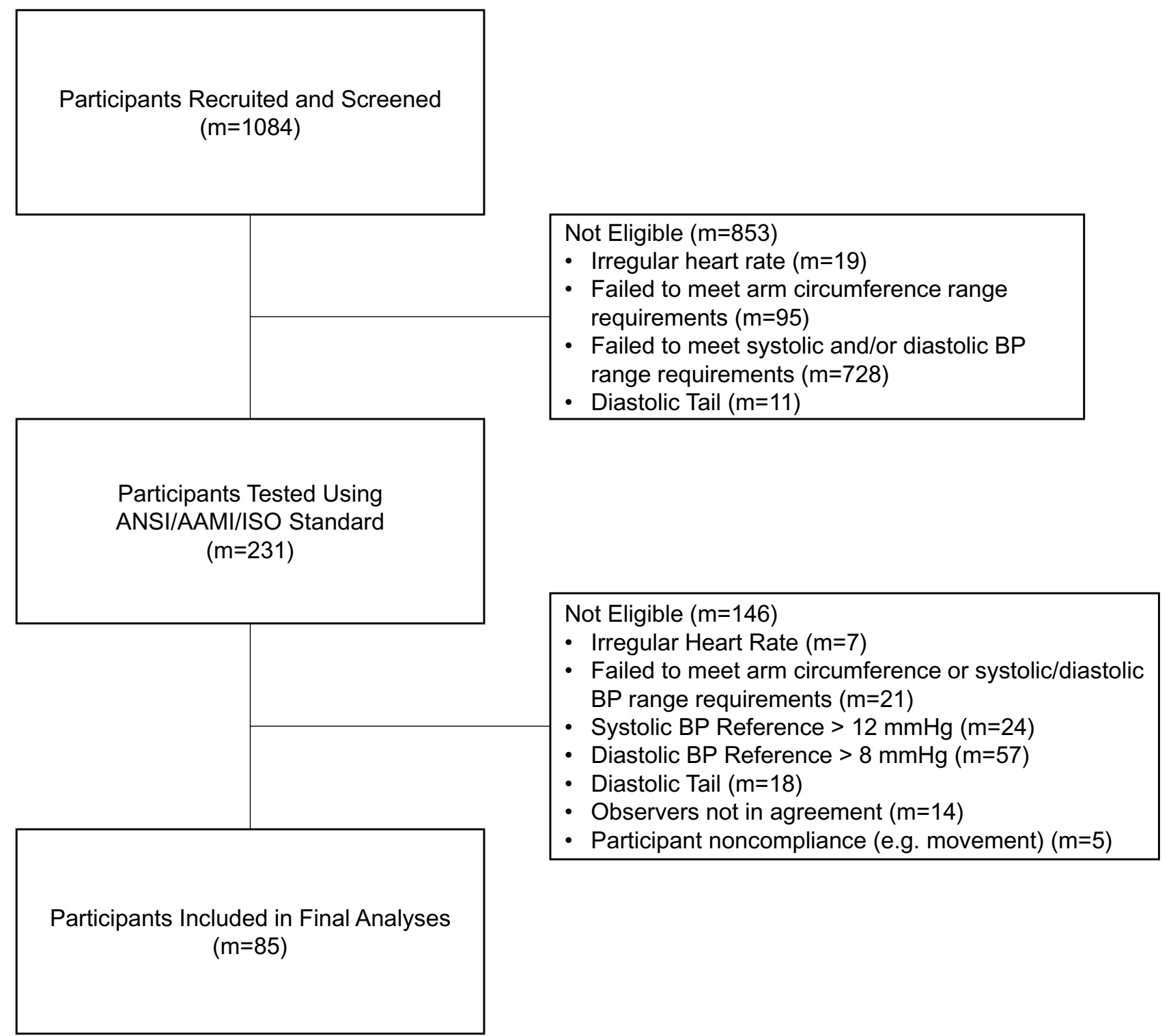

Figure 2 Flow diagram of participants through recruitment, testing, and analysis. The 85 participants included in the final analyses each had 3 paired determinations considered valid according to ANSI/AAMI/ISO 81060-2:20I3 standards. 
Table I Participant Characteristics and ANSI/AAMI/ISO 81060-2:2013 Requirements

\begin{tabular}{|c|c|c|}
\hline & Required & Actual \\
\hline Age* & $>12$ years & $\begin{array}{l}36.8 \pm 14.0,13-73 \\
\text { years }\end{array}$ \\
\hline Height* & N/A & $\begin{array}{l}167.9 \pm 10.1 \\
147-188 \mathrm{~cm}\end{array}$ \\
\hline Weight* & $N / A$ & $\begin{array}{l}82.1 \pm 21.9 \\
45-132 \mathrm{~kg}\end{array}$ \\
\hline $\begin{array}{l}\text { Number of qualified } \\
\text { participants }\end{array}$ & $\mathrm{m} \geq 85$ & $\mathrm{~m}=85$ \\
\hline $\begin{array}{l}\text { Number of determination } \\
\text { pairs }(3 \times 85)\end{array}$ & $n \geq 255$ & $\mathrm{n}=255$ \\
\hline \multirow[t]{2}{*}{ Gender** } & $30 \%$ Male & 37 (43.5\%) Males \\
\hline & $30 \%$ Female & $48(56.5 \%)$ Females \\
\hline \multirow{4}{*}{$\begin{array}{l}\text { Arm circumference** } \\
\text { (Range: } 24-44 \mathrm{~cm} \text { ) }\end{array}$} & $40 \%$ Upper half & $4 \mathrm{I}(48.2 \%) \geq 34 \mathrm{~cm}$ \\
\hline & $40 \%$ Lower half & $45(52.9 \%)<34 \mathrm{~cm}$ \\
\hline & $20 \%$ Upper quarter & $19(22.4 \%) \geq 39 \mathrm{~cm}$ \\
\hline & $20 \%$ Lower quarter & $19(22.4 \%)<29 \mathrm{~cm}$ \\
\hline \multirow[t]{3}{*}{ Systolic BP** } & $5 \% \geq 160 \mathrm{mmHg}$ & $5(5.9 \%)$ \\
\hline & $5 \% \leq 100 \mathrm{mmHg}$ & $12(14.1 \%)$ \\
\hline & $20 \% \geq 140 \mathrm{mmHg}$ & $17(20.0 \%)$ \\
\hline \multirow[t]{3}{*}{ Diastolic BP** } & $5 \% \geq 100 \mathrm{mmHg}$ & $5(5.9 \%)$ \\
\hline & $5 \% \leq 60 \mathrm{mmHg}$ & $9(10.6 \%)$ \\
\hline & $20 \% \geq 85 \mathrm{mmHg}$ & $23(27.1 \%)$ \\
\hline
\end{tabular}

Notes: All participant characteristic requirements for the ANSI/AAMI/ISO 81060-2:2013 standards (clinical investigation of the automated measurement type) were met. BP, blood pressure. *Mean \pm SD, Range. **Number (and percentage) of participants.

placed. Cuff size for manual measurements was determined by measuring arm circumference following the National Health and Nutrition Examination Survey (NHANES) upper arm circumference method and selecting the appropriate cuff using the AHA guidelines. ${ }^{15}$ The individual was seated comfortably and was asked not to talk or move for the entire duration of the data collection period, with 5 minutes of resting quietly before BP testing began.

\section{Instrumentation}

Reference BP measurements were simultaneously determined by two trained observers experienced in the use of the manual (auscultatory) method with a double stethoscope. The observers completed the British Hypertension Society's online training videos and read the required AHA article about recommendations for BP measurements in humans. ${ }^{15}$ A National Institute of Standards and Technology (NIST) calibrated mercury gravity sphygmomanometer (ADC 972 Diagnostix, Hauppauge, NY) was used as the reference standard. The certified device has a range from $0 \mathrm{mmHg}$ to 300 $\mathrm{mmHg}$ with proper unit division on the tube. Observers made simultaneous BP determinations on each subject using a double Littman ${ }^{\circledR}$ stethoscope (3M, St. Paul, MN). The peak inflation level was determined by adding a minimum of $30 \mathrm{mmHg}$ to the pressure at which the palpated radial artery pulse was eliminated on inflation of the cuff. Each observer's recording of observations of the reference sphygmomanometer were not visible to the other observer. Each reference BP determination was calculated as the average of the two observers' blinded values. Any pair of observers' determinations with a difference greater than $4 \mathrm{mmHg}$ was excluded. Observers were notified after each reading whether this requirement was met.

The ABPS was operated by a third study investigator. This USC overseer was trained in proper use of the ABPS, recording data, and ensuring that the two observers were blinded to each other's measured values and those of the ABPS. A schematic diagram of the ABPS, including the new mechanical cuff on a sit-down health kiosk, is shown in Figure 1. Using US anthropometric data, ${ }^{16}$ the ABPS is designed to measure BP accurately in an arm circumference range $(24-44 \mathrm{~cm})$ that covers approximately $\geq 95 \%$ of US adult females and males. The patent for this system (US 2018/0184924 A1) is held by higi SH 1lc. ${ }^{13}$ First, an adapted knuckle support assembly provides the cuff with four degrees freedom of movement (ie, forward/backward translation, left/right translation, up/down translation, and rotation) for postural positioning before the BP assessment. This allows the participant to sit comfortably in their natural upright position, regardless of their body size. Note that this knuckle support assembly moves independently of the ABPS's electrical components. With the participant seated comfortably, legs uncrossed, feet flat on the floor, and their left arm in the cuff with elbow and forearm supported, the middle of the cuff naturally sits at the level of the right atrium of the heart. The study investigator initiated the system only when all these criteria were met.

Upon initiation, the ABPS activates a motorized gearhead that automatically wraps the cuff down to the user's arm prior to inflation. The cuff's bladder then inflates to approximately $160 \mathrm{mmHg}$ pressure and inflates higher if a systolic pulse is detected at the initial inflate value. A previously validated ${ }^{17}$ SunTech BP module is integrated 
within the ABPS to control inflation, deflation, and pulse detection: Advantage Module 2.0 (LX3.413) and SafetyMicroRev (SMV221). The module was calibrated against an NIST certified Omega Digital Manometer (Omega Engineering INC., Norwalk, CT) over a range from 40 to $260 \mathrm{mmHg}$ prior to all testing. By wrapping the cuff down to the user's arm prior to inflation, the volume of air in the bladder during measurements is decreased, thus improving the signal-to-noise ratio. This mechanism also provides a measure of arm circumference through an algorithm that estimates how much the cuff wraps down. Once the BP assessment is completed, the cuff fully deflates and expands, returning to the circumference of the starting position. The user interface displays the ABPS's measurements of systolic BP, diastolic BP, heart rate, and arm circumference.

\section{Study Design}

Following the ANSI/AAMI/ISO 81060-2:2013 standard, ${ }^{14}$ observers first determined the participant's BP using the reference sphygmomanometer. The reference sphygmomanometer was then removed, and the participant placed their arm in the ABPS. Arm positioning for the reference and ABPS measurements were all at the same height level (ie, heart level) so participants could remain seated in the kiosk health station. After 60 seconds of rest, the participant's BP was determined using the ABPS. Data from these initial measurements were used for baseline purposes only, and not included in the statistical analyses. Subsequent BP assessments were eligible for statistical analyses, performed in the following order. Observers again determined the participant's BP using the reference sphygmomanometer. Then, the team sequentially determined BP alternating between the ABPS and reference sphygmomanometer until the required number of determinations had been performed. Rest periods between all determinations were no less than 60 seconds.

Three valid pairs of determinations were required for each participant to be included in statistical analyses. Each paired determination consisted of one ABPS BP measurement and the average of the reference BP measurements immediately before and after the ABPS measurement. The following criteria needed to be met for a paired determination to be considered valid. All participant data exclusion decisions during and after testing were made by the blinded observers without knowledge of each other's readings or that of the ABPS (Figure 2). Any reference determination with a difference between observers greater than
$4 \mathrm{mmHg}$ was excluded, along with the corresponding ABPS BP measurement. A maximum of 8 paired determinations could be performed to reach 3 valid pairs. If 3 valid pairs of determinations could not be obtained, all the participant's data were excluded. All data from a participant were also excluded if any two valid reference systolic BP determinations differed by more than 12 $\mathrm{mmHg}$ or if any two valid reference diastolic BP determinations differed by more than $8 \mathrm{mmHg}$.

\section{Statistics}

Validation of the ABPS was determined by the requirements set forth by the ANSI/AAMI/ISO 81060-2:2013 standard. Data collection continued until 85 participants met the participant characteristic requirements with 3 valid paired BP determinations. For the device to pass the standard's statistical requirements and be considered an accurate device for measuring $\mathrm{BP}$, it needs to meet the following two criteria. Criterion 1: for systolic and diastolic BP, the mean value of the differences of all 255 valid paired determinations $(\overline{\mathrm{X}} \mathrm{n})$ shall be within or equal to \pm 5 $\mathrm{mmHg}$ with a standard deviation (Sn) no greater than 8 $\mathrm{mmHg}$. These mean difference values for systolic and diastolic BP represent the mean error between ABPS BP measurements and reference BP measurements from 255 paired assessments. Criterion 2: the 3 valid paired determinations for each participant were averaged, producing 85 averaged paired determinations. For systolic and diastolic BP, the standard deviation of the averaged paired determinations $(\mathrm{Sm})$ shall meet the criteria listed in the standards. The maximum permissible standard deviation $(\mathrm{Sm})$ is a function of mean difference $(\overline{\mathrm{X}} \mathrm{n})$ calculated in Criterion 1.

Additional analyses were performed to explore the variables that influence $\mathrm{BP}$ and arm circumference measurements by the ABPS. The following are not part of the ANSI/AAMI/ISO 81060-2:2013 standard protocols. First, data from the 85 participants included in Criterions 1 and 2 were used in linear regression analyses to explore the effects of NHANES arm circumference and body mass index (BMI) on BP difference. In addition, participants were given a Likert scale to assess the level of comfort associated with the ABPS BP measurements compared to the manual fitted cuff method after BP data collection. Responses are summarized in the results section.

Two hundred and seventeen participants had at least three ABPS arm circumference measurements. All analyses with ABPS arm circumference used the average of 
these three measurements for each participant. The 217 ABPS arm circumferences were compared to the NHANES method to determine device accuracy using a paired $t$-test. Alpha level was set at 0.05 (IBM SPSS Statistics Version 25). Linear regression analysis was performed to explore the effects of BMI on arm circumference difference (the difference between the NHANES arm circumference measurement and ABPS arm circumference measurement). Last, a sequential regression analysis was performed with a split-sample (80/20) to generate a prediction model for NHANES arm circumference using only one predictor, the ABPS's arbitrary units. Participant characteristics were then tested to improve the model's performance. To be retained in the final model, a component had to be statistically significant and account for at least $1 \%$ of the variance in NHANES arm circumference.

\section{Results}

All enrollment criteria within the ANSI/AAMI/ISO 81060-2:2013 standard were met by the study. The disappearance of the Korotoff sound (K5) was used on 82 participants, while K4 was used on 3 participants. For systolic and diastolic BP, the mean paired determination difference and standard deviation of the difference (Criterion 1) were within the requirements (Table 2). Similarly, the standard deviations of the averaged paired determination differences (Criterion 2) were within the maximum permissible values (Table 2).

The systolic and diastolic Bland-Altman plots show the distribution of all 255 paired determination differences as a function of the mean BP between ABPS and reference

Table 2 Validation Results for ANSI/AAMI/ISO 81060-2:2013

\begin{tabular}{|l|l|l|}
\hline Criterion I (n = 255) & $\begin{array}{l}\text { Mean Difference } \\
(\overline{\mathbf{X}} \mathbf{n})\end{array}$ & $\begin{array}{l}\text { Standard } \\
\text { Deviation (Sn) }\end{array}$ \\
\hline Systolic Pressure $(\mathrm{mmHg})$ & -2.4 & 7.7 \\
Diastolic Pressure $(\mathrm{mmHg})$ & 1.7 & 5.7 \\
\hline Criterion 2 (m = 85) & Standard & Maximum \\
& Deviation $\mathbf{( S m )}$ & Permissible (Sm) \\
\hline Systolic Pressure $(\mathrm{mmHg})$ & 6.3 & 6.5 \\
Diastolic Pressure $(\mathrm{mmHg})$ & 5.2 & 6.7 \\
\hline
\end{tabular}

Notes: Both criterions set forth by the ANSI/AAMI/ISO 81060-2:2013 standards were met for validation of the ABPS. Criterion I: the mean value of the differences of all 255 valid paired determinations $\left(\bar{X}_{n}\right)$ needed to be less than or equal to \pm 5 $\mathrm{mmHg}$ with a standard deviation $(\mathrm{Sn})$ no greater than $8 \mathrm{mmHg}$. Criterion 2: The standard deviation of the averaged paired determinations per participant $(\mathrm{Sm})$ needed to meet the criteria listed in the standards as a function of mean difference calculated in Criterion I (X̄ $n)$.
(Figure 3). The systolic BP differences in Figure 3A appeared to be evenly distributed despite some outliers. The device tended to slightly overestimate diastolic BP at low values $(<60 \mathrm{mmHg})$ and underestimate at high values $\quad(>100$ $\mathrm{mmHg}$ ), as shown in Figure 3B. These slight offsets did not affect the overall passing results.

The mean NHANES arm circumference of the 85 participants that qualified for inclusion in the validation portion of the study was $33.8 \mathrm{~cm}$ (range: $24-44 \mathrm{~cm}$ ). Plots were made using the average BP difference for each participant as a function of their NHANES arm circumference (Figure 4A and B). Data for the systolic and diastolic BP distribution showed no evidence of a systematic under- or over-estimation for the range of arm circumferences measured. The effects of BMI on ABPS accuracy were also analyzed this way, but for both systolic and diastolic BP measurements there was no systemic effect of BMI on BP difference. However, there appeared to be greater variability at BMIs $>30 \mathrm{~kg} / \mathrm{m}^{2}$ for a few individuals.

Of the 217 participants with a minimum of 3 arm circumference measurements, the average of three ABPS measurements had a strong correlation with the NHANES arm circumference measurements $\left(\mathrm{R}^{2}=0.87\right.$, Slope $=$ 0.75 , Intercept $=9.77$, Figure 5A). The mean difference $(\overline{\mathrm{X}} \mathrm{c})$ between ABPS arm circumference and NHANES arm circumference and the standard deviation (Sc) of the difference were small $(-2.06 \pm 2.36 \mathrm{~cm})$. The arm circumference Bland-Altman plot shows the distribution of all paired differences as a function of the mean arm circumference between ABPS and NHANES (Figure 5B). This plot suggests that the ABPS tends to underestimate arm circumference at smaller arm sizes $(\mathrm{r}=0.34, \mathrm{p}<0.01)$.

Sequential regression analysis was performed on a training dataset of 173 randomly selected participants to generate prediction models for NHANES arm circumference. Validation was performed on a holdout dataset of the remaining 44 participants. The first model was made using only the ABPS's arbitrary units. Although the linear model (Model 1) was significant $\left(\mathrm{R}^{2}=0.86\right)$, adding the secondorder term increased $\mathrm{R}^{2}$ by a significant 0.015 , so the quadratic model was justified as being superior. Adding the third-order term did not increase $\mathrm{R}^{2}$ so we chose to reject the cubic model to avoid over-fitting. The quadratic model (Model 2) was strong in both training $\left(\mathrm{R}^{2}=0.88\right)$ and validation $\left(\mathrm{R}^{2}=0.89\right)$ datasets. Of the participant characteristics collected, BMI was the strongest predictor variable. To improve its distribution, the inverse of BMI was used. Adding the inverse of BMI increased $\mathrm{R}^{2}$ of Model 2 by 
A

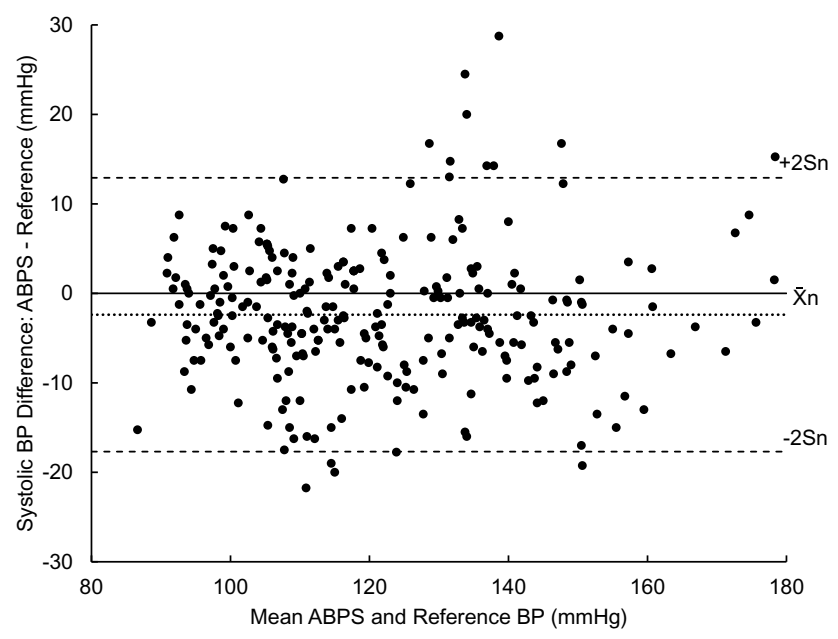

B

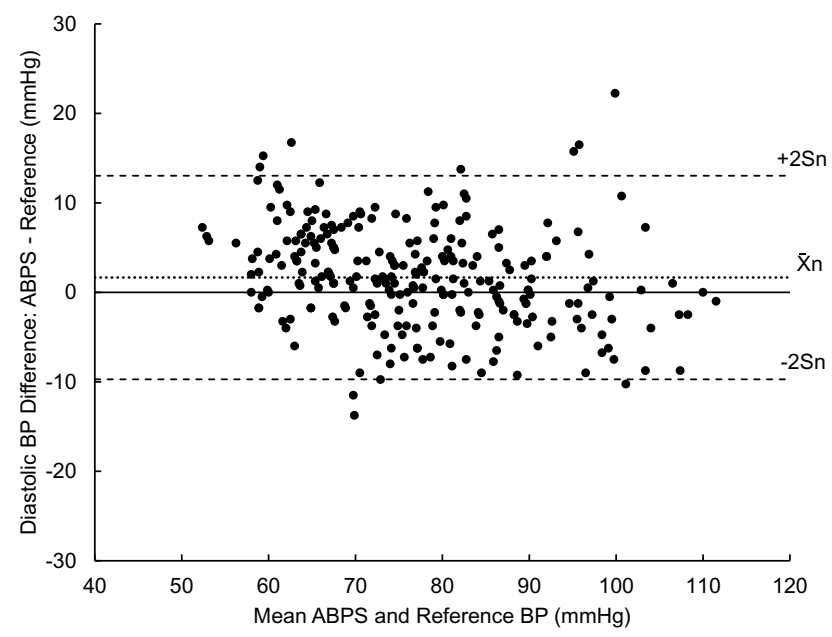

Figure 3 Bland-Altman plots of 255 paired determinations $(n=255)$. (A) Systolic pressures: differences versus means, (B) Diastolic pressures: differences versus means. Differences were calculated by subtracting valid ABPS BP measurements by their corresponding reference BP measurements. Means were calculated using valid ABPS BP measurements and their corresponding reference BP measurements. Indications for mean (X̄n) $\pm 2 \mathrm{Sn}$ are shown.

A

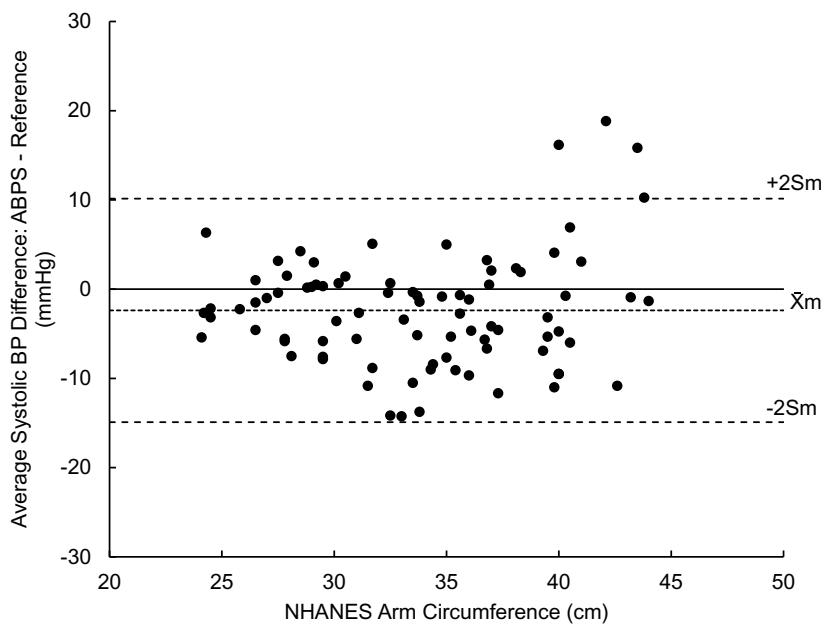

B

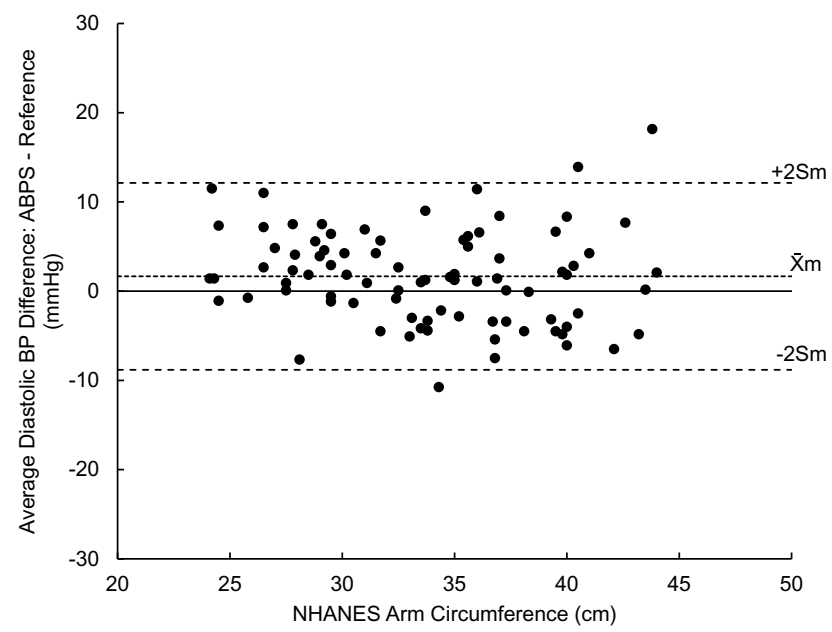

Figure 4 Plots of mean $(\mathbf{A})$ systolic and $(B)$ diastolic BP difference versus NHANES arm circumference $(\mathrm{m}=85)$. Differences were calculated by subtracting valid ABPS BP measurements by their corresponding reference BP measurements. The average of three BP differences for each of the 85 participants is plotted against the participant's arm circumference measured by the NHANES method. Indications for mean $(\overline{\mathrm{X}} \mathrm{m}) \pm 2 \mathrm{Sm}$ are shown.

a significant 0.054 . This model (Model 3) was also strong in both training $\left(\mathrm{R}^{2}=0.93\right.$, Difference $\left.=0.01 \pm 1.31 \mathrm{~cm}\right)$ and validation $\left(\mathrm{R}^{2}=0.94\right.$, Slope $=0.99$, Intercept $=0.24$, Difference $=0.32 \pm 1.24 \mathrm{~cm}$, Figure 6A and B) datasets.

After BP testing, participants were presented with the following statement to assess ABPS comfort: "The level of comfort when using the ABPS is equal to or better than the manual fitted cuff method", and asked to provide their answer: "1-Strongly Disagree" (3 Participants), "2-Disagree" (15 Participants), "3-Neutral" (36 Participants), "4-Agree" (85 Participants), "5-Strongly Agree" (72 Participants). The median and mode response was "4-Agree", with a mean and standard deviation of $4.1 \pm 1.0$.

\section{Discussion}

The ABPS has met all ANSI/AAMI/ISO 81060-2:2018 "Non-invasive sphygmomanometers - Part 2: Clinical investigation of automated measurement type" requirements using the same arm sequential method. Furthermore, the standard recommends using more than one testing site and device, ${ }^{14}$ which the present study achieved as part of its design (4 locations, 2 devices). These standards ensure the quality of 
A

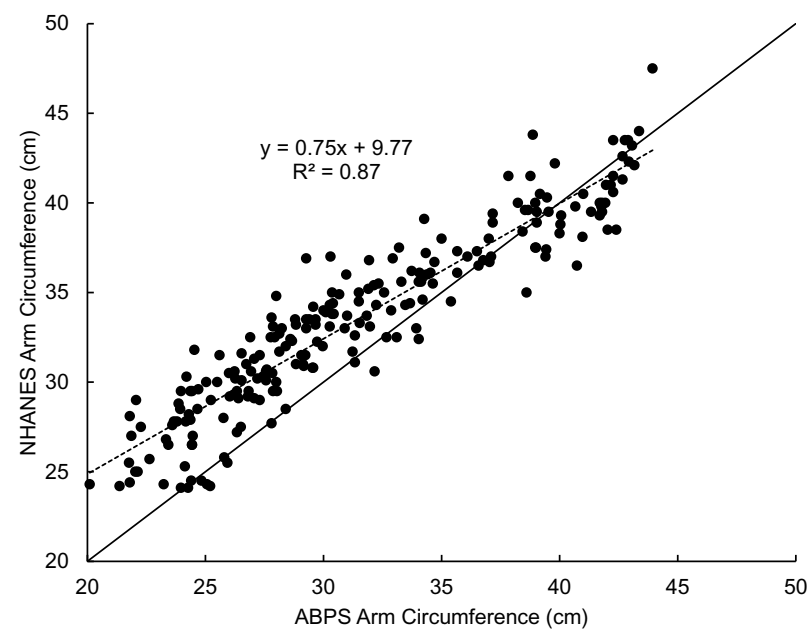

B

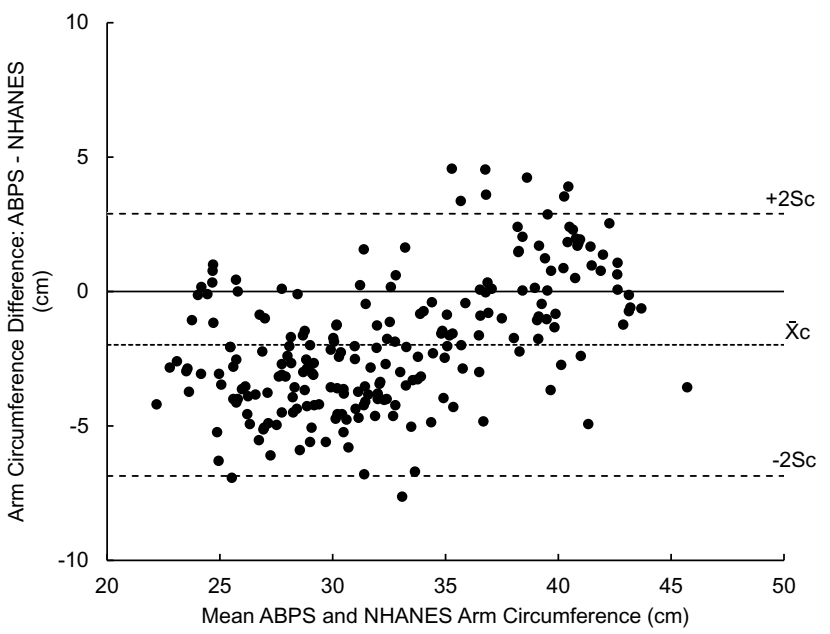

Figure 5 Bland-Altman plots of 217 participants' arm circumference. (A) NHANES arm circumference is plotted against the average of three ABPS arm circumference measurements. A reference line $(y=x)$ is included. (B) Bland-Altman plots of ABPS arm circumference difference versus mean of ABPS and NHANES. Differences were calculated by subtracting the average of three ABPS arm circumference measurements by their corresponding NHANES measurement. Indications for mean (X) \pm 2 Sc are shown.

A

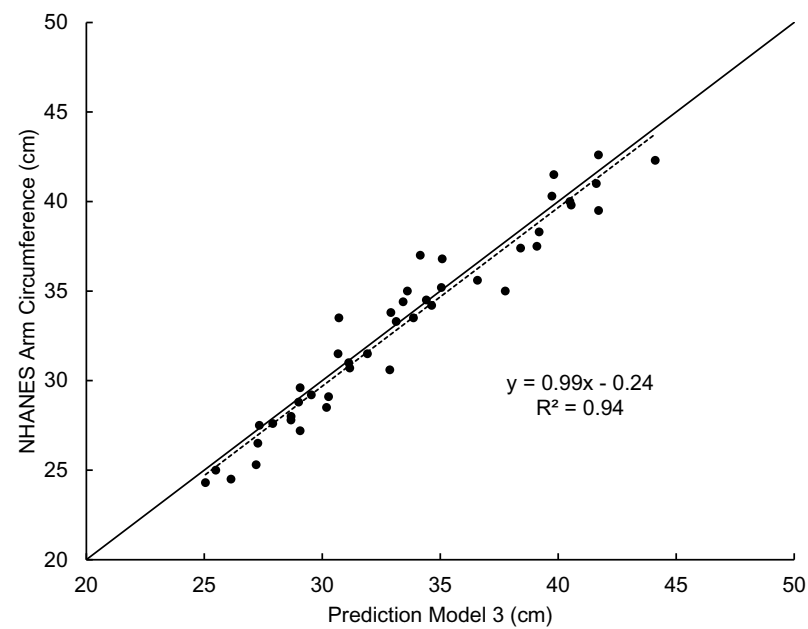

B

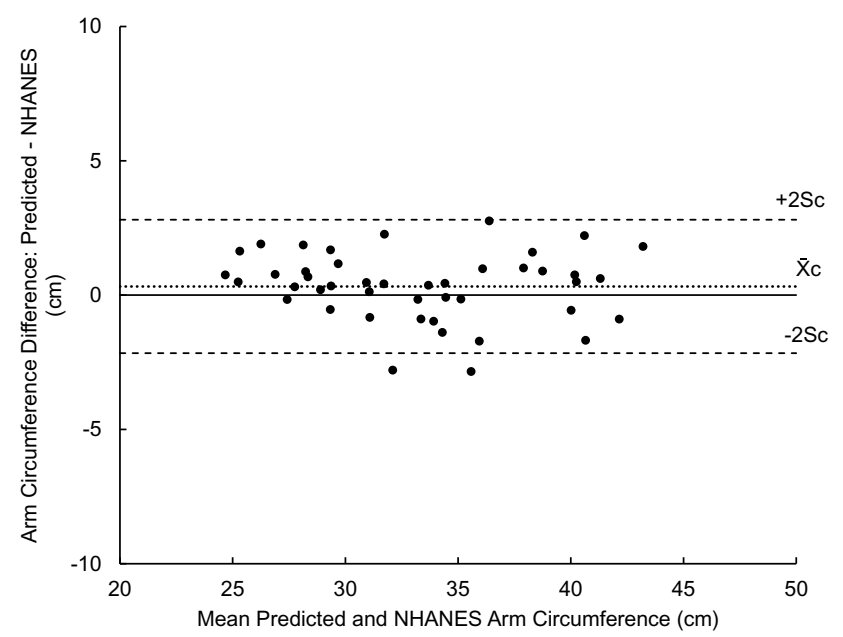

Figure 6 New prediction model (Model 3) accuracy for 44 participants in the holdout dataset. (A) NHANES arm circumference is plotted against the Model 3 predicted arm circumferences. A reference line $(y=x)$ is included. (B) Bland-Altman plots of predicted arm circumference difference versus mean of predicted and NHANES. Differences were calculated by subtracting the Model 3 predicted arm circumferences by their corresponding NHANES measurements. Indications for mean $(\bar{X} \mathrm{c}) \pm 2 \mathrm{Sc}$ are shown.

validation studies and reduce the risk of reporting false conclusions. Adherence to these standards is extremely important, as inaccurate readings can have significant clinical implications. $^{10}$

The ABPS was designed to improve the range, accuracy, and comfort of use with the higi health station. To the best of the authors' knowledge, this is the first validated public health kiosk BP cuff to use an automated gearhead that wraps the cuff down to the user's arm circumference prior to initiation of BP measurement, reducing noise to signal ratio and leading to improved accuracy. ${ }^{13}$ In addition, the arm support includes an adapted knuckle assembly to provide several degrees of freedom of movement that facilitates optimal positioning and allows the user to relax their arm for more accurate readings. We believe this provides significant benefits to real-world BP measurements.

The results are consistent with previous studies, which is unsurprising given that the SunTech BP module integrated within the ABPS's cuff was previously validated using the 2003 AAMI standards. ${ }^{17}$ The mean difference 
and standard deviation of the difference for the SunTech BP Module (systolic: $1.7 \pm 3.1 \mathrm{mmHg}$, diastolic: $1.1 \pm 3.2$ $\mathrm{mmHg}$ ) were slightly lower than our results for the ABPS. However, validation studies of automated kiosks typically have similar findings as reported here; for example, the Pharma-Smart PS-2000 ${ }^{18}$ (systolic: $0.7 \pm 7.0 \mathrm{mmHg}$, diastolic: $-0.3 \pm 6.6 \mathrm{mmHg}$ ) and the Computerized Screening Inc Health Station $6 \mathrm{~K}^{19}$ (systolic: $1.5 \pm 5.8 \mathrm{mmHg}$, diastolic: $0.5 \pm 4.4 \mathrm{mmHg}$ ). These earlier versions of the AAMI standards did not require a Criterion 2 analysis; thus, we could not compare to the present results.

This study validates the ABPS in a wide range of body and arm sizes. The slight effect of BMI on the accuracy of BP and arm circumference measurements is a common and important issue with BP cuffs, as obesity is a major risk factor for hypertension. ${ }^{6}$ These results further emphasize the importance of strict participant characteristic requirements in validation protocols. The ANSI/AAMI/ISO standard requires even distributions of arm circumference, ${ }^{14}$ whereas the European Hypertension Society-International Protocol does not have such requirements, ${ }^{20}$ which can often result in a bias toward testing primarily standard adult arm circumferences and not small or large sizes. Figure 4 does not show a significant effect of arm circumference on systolic or diastolic BP difference. These results place greater confidence in the validated arm circumference range. In practice, the cuff may be able to accommodate an even wider range since the cuff housing circumference is $\sim 46 \mathrm{~cm}$ ( 18 inches).

Arm circumference measurements are also unique to this device compared to other BP cuffs. The arm circumference analyses are not part of the ANSI/AAMI/ISO 81060-2:2013 standards and therefore do not affect the validation of the ABPS. However, these analyses did demonstrate the accuracy of arm circumference measurements by the ABPS and helped develop a new algorithm for improved accuracy in future measurements. Measurements were within an acceptable tolerance using the preliminary model, despite a slight underestimation of arm circumference with smaller arm sizes reported in Figure 5. We used this data to generate a new prediction model for arm circumference. The new model correctly explained $94.3 \%$ of the variance in arm circumference of a holdout dataset. Updating the algorithm will allow the ABPS to produce accurate measurements that can be tracked over time and help clinicians choose the appropriate cuff size for subsequent manual BP assessments.
This study validates the ABPS for use in relatively healthy populations, without known arrhythmias or current pregnancy. However, in practice, these kiosks are fully automated for public use where instructions for correct posture and operation during BP self-measurement are not always followed appropriately. Therefore, special precautions are warranted to encourage compliance in order to obtain BP measurements of good quality. Comfort, in particular, can improve compliance. Most participants in this study rated the comfort of the ABPS to be equal to or better than the manual fitted cuff.

In conclusion, the ABPS measures BP accurately on a wide range of arm circumferences $(24-44 \mathrm{~cm}$ ) based on the comparison to the auscultatory method. Precision was demonstrated by repeated measurements. Therefore, this study affirms the usefulness of the ABPS in assisting health-care personnel to better monitor BP away from the office and inform better medical management of patients with hypertension.

\section{Data Sharing Statement}

Individual-deidentified participant data and study-related documents will not be made available.

\section{Acknowledgments}

This study was funded by higi SH llc. We thank all study participants as well as the South Pasadena Senior Center and the Pasadena Department of Public Health for their participation. In addition, we thank the following individuals for their contributions to this time-intensive study: Emily Garcia, Malcolm Jones, Yunae Lee, Emmanuel Lavarias, Katie Lerch.

\section{Disclosure}

Mr Timothy R Macaulay reports grants from Stayhealthy Inc, during the conduct of the study. David N. Erceg and Bjorn McMillan were fulltime employees of Stayhealthy Inc., contracted by higi SH 1lc for the duration of the study to provide BP device operational support and aid in the collection of study data. Both served as reference BP observers and were completely blinded to the readings of each other and the Automated Blood Pressure System during testing. David N. Erceg was contracted for the duration of the study, while Bjorn McMillan was only contracted for $50 \%$. Both also have a patent US20180184924A1 pending. The authors report no other conflicts of interest in this work. 


\section{References}

1. Abubakar I, Tillmann T, Banerjee A. Global, regional, and national age-sex specific all-cause and cause-specific mortality for 240 causes of death, 1990-2013: a systematic analysis for the Global Burden of Disease Study 2013. Lancet. 2015;385(9963):117-171.

2. Lim SS, Vos T, Flaxman AD, et al. A comparative risk assessment of burden of disease and injury attributable to 67 risk factors and risk factor clusters in 21 regions, 1990-2010: a systematic analysis for the Global Burden of Disease Study 2010. Lancet. 2012;380 (9859):2224-2260. doi:10.1016/S0140-6736(12)61766-8

3. Huang Y, Wang S, Cai X, et al. Prehypertension and incidence of cardiovascular disease: a meta-analysis. BMC Med. 2013;11(1):177. doi:10.1186/1741-7015-11-177

4. Ford ES. Trends in mortality from all causes and cardiovascular disease among hypertensive and nonhypertensive adults in the United States Clinical perspective. Circulation. 2011;123 (16):1737-1744. doi:10.1161/CIRCULATIONAHA.110.005645

5. Egan BM, Li J, Hutchison FN, Ferdinand KC. Hypertension in the United States 1999-2012: progress toward healthy people 2020 goals. Circulation. 2014;130(19):1692-1699. doi:10.1161/ CIRCULATIONAHA.114.010676

6. Whelton PK, Carey RM, Aronow WS, et al. 2017 ACC/AHA/AAPA/ $\mathrm{ABC} / \mathrm{ACPM} / \mathrm{AGS} / \mathrm{APhA} / \mathrm{ASH} / \mathrm{ASPC} / \mathrm{NMA} / \mathrm{PCNA}$ guideline for the prevention, detection, evaluation, and management of high blood pressure in adults: a report of the American College of Cardiology/ American Heart Association Task Force on clinical practice guidelines. J Am Coll Cardiol. 2018;71(19). e127-e248.

7. Muntner P, Carey RM, Gidding S, et al. Potential US population impact of the 2017 ACC/AHA high blood pressure guideline. Circulation. 2018;137(2):109-118. doi:10.1161/CIRCULATIONAHA.117.0325 82

8. Ioannidis JP. Diagnosis and treatment of hypertension in the 2017 ACC/AHA guidelines and in the real world. JAMA. 2018;319 (2):115-116. doi:10.1001/jama.2017.19672

9. Houle SK, Chuck AW, Tsuyuki RT. Blood pressure kiosks for medication therapy management programs: business opportunity for pharmacists. J Am Pharm Assoc. 2012;52(2):188-194. doi:10.1331/ JAPhA.2012.11217
10. Al Hamarneh YN, Houle SK, Chatterley P, Tsuyuki RT. The validity of blood pressure kiosk validation studies: a systematic review. Blood Press Monit. 2013;18(3):167-172. doi:10.1097/MBP.0b013e328360fb85

11. Alpert BS, Dart RA, Sica DA. Public-use blood pressure measurement: the kiosk quandary. J. Am. Soc. Hypertens. 2014;8 (10):739-742. doi:10.1016/j.jash.2014.07.034

12. Banner TE, Gravenstein J. Comparative effects of cuff size and tightness of fit on accuracy of blood pressure measurements. $J$ Clin Monit. 1991;7(4):281-284. doi:10.1007/BF01619345

13. McMillan B, Erceg D, Flammer JD, Nunez R, Hill CK, Collins JR. Automated fitted cuff blood pressure and arm circumference measuring device. Google Patents; 2018.

14. Association for the Advancement of Medical Instrumentation. NonInvasive Sphygmomanometers-Part 2: Clinical Validation of Automated Measurement Type. Arlington, VA: American National Standard; 2013.

15. Pickering TG, Hall JE, Appel LJ, et al. Recommendations for blood pressure measurement in humans and experimental animals: part 1: blood pressure measurement in humans: a statement for professionals from the Subcommittee of Professional and Public Education of the American Heart Association Council on High Blood Pressure Research. Circulation. 2005;111(5):697-716. doi:10.1161/01.CIR.0000154900.76 284.F6

16. Fryar CD, Gu Q, Ogden CL, Flegal KM. Anthropometric reference data for children and adults; United States, 2011-2014. 2016.

17. Friz HP, Facchetti R, Primitz L, et al. Simultaneous validation of the SunTech 247 diagnostic station blood pressure measurement device according to the British Hypertension Society protocol, the International Protocol and the Association for the Advancement of Medical Instrumentation standards. Blood Press Monit. 2009;14 (5):222-227. doi:10.1097/MBP.0b013e328330c873

18. Alpert BS. Validation of the pharma-smart PS-2000 public use blood pressure monitor. Blood Press Monit. 2004;9(1):19-23. doi:10.1097/ 00126097-200402000-00005

19. Buxton IL, Adams JQ, Gore M, Sullivan CR. Validation of the CSI health station $6 \mathrm{~K}$ blood pressure Kiosk ${ }^{\circledR}$. Paper Presented at: Proceedings of the Western Pharmacology Society;2007.

20. O'Brien E, Atkins N, Stergiou G, et al. European society of hypertension international protocol revision 2010 for the validation of blood pressure measuring devices in adults. Blood Press Monit. 2010;15(1):23-38. doi:10.1097/MBP.0b013e3283360e98
Vascular Health and Risk Management

\section{Publish your work in this journal}

Vascular Health and Risk Management is an international, peerreviewed journal of therapeutics and risk management, focusing on concise rapid reporting of clinical studies on the processes involved in the maintenance of vascular health; the monitoring, prevention and treatment of vascular disease and its sequelae; and the involvement of metabolic disorders, particularly diabetes. This journal is indexed on PubMed Central and MedLine. The manuscript management system is completely online and includes a very quick and fair peerreview system, which is all easy to use. Visit http://www.dovepress. com/testimonials.php to read real quotes from published authors. 